\title{
Stability and Structure of Inclusion Complexes of Zaleplon with Natural and Modified Cyclodextrins ${ }^{\dagger}$
}

\author{
Jasna Jablan, Tin Weitner, Mario Gabričević,* and Mario Jug \\ Faculty of Pharmacy and Biochemistry, University of Zagreb, A. Kovačića 1, 10000 Zagreb, Croatia
}

RECEIVED NOVEMBER 23, 2010; REVISED MARCH 15, 2011; ACCEPTED MARCH 17, 2011

\begin{abstract}
The interaction between zaleplon (ZAL) and different cyclodextrins in aqueous solutions was investigated by spectrofluorimetric and phase solubility studies. Stability constants determined by both methods showed that among natural cyclodextrins, $\beta$-cyclodextrin $(\beta C D)$ formed the most stabile complex but its solubilizing efficiency was limited. Among $\beta C D$ derivatives, the complex stability and solubilisation efficiency decreased in order: randomly methylated- $\beta C D$ (RAMEB) $>$ sulphobutylether- $\beta C D$ $(\mathrm{SBE} \beta \mathrm{CD})>$ hydroxypropyl- $\beta \mathrm{CD}$ (HP $\beta C D)$. The inclusion complexes of ZAL with $\beta C D$ and RAMEB were further characterised by ${ }^{1} \mathrm{H}-\mathrm{NMR}$ spectroscopy and the inclusion complex formation was confirmed in both cases. ROESY spectra showed two binding modes between ZAL and $\beta C D$ which exist simultaneously in the solution. The first binding mode occurs by the inclusion of the phenyl ring of ZAL into the $\beta C D$ central cavity via the wider rim of the cyclodextrin cone and is dominant. The second one is formed by the inclusion of pyrazolo[1,5-a]pyrimidine ring of ZAL.(doi: 10.5562/cca1800)
\end{abstract}

Keywords: Cyclodextrins, Zaleplon, Inclusion Complexes, Stability Constants, Fluorescence, ${ }^{1} \mathrm{H}-\mathrm{NMR}$

\section{INTRODUCTION}

Zaleplon (ZAL), $N$-[3-(3-cyanopyrazolo[1,5-a]py-rimidin-7-yl)phenyl]- $N$-ethylacetamide, is a nonbenzodiazepine hypnotic drug of the pyrazolopyrimidine class. In the dose of 5 to $10 \mathrm{mg}$, the drug is indicated for the short term management of insomnia. ${ }^{1,2}$ ZAL also shows potent anticonvulsant activity against electroshock-induced convulsions. ${ }^{3}$ The drug is a lipophilic compound which is virtually insoluble in water. ${ }^{4}$ It has been recognised that a certain level of aqueous solubility is required of the drug substance to be readily delivered to the cellular membrane, but at the same time it has to be lipophilic enough to cross the membrane itself. In the case of lipophilic compounds such as ZAL, the dissolution in gastrointestinal media is often a ratelimiting step for the absorption, thus limiting the oral bioavailability of the drug. ${ }^{5}$ In the liver, ZAL is extensively metabolized into pharmacologically inactive metabolites. ${ }^{3}$ Low aqueous solubility of ZAL and its significant presystemic metabolism lead to a rather low oral bioavailability of about $30 \%{ }^{6}$

Various formulation techniques can be applied to overcome the low aqueous solubility of drugs without affecting their optimised pharmacological action. Among different approaches for enhancing the aqueous solubility of lipophilic drugs, cyclodextrin (CD) complexation proved to be one of the most effective. ${ }^{7}$ Cyclodextrins are a group of structurally related oligosaccharides, consisting of 6,7 or $8(\alpha-1,4)$-linked $\alpha$-D-glucopiranose units $(\alpha-, \beta$ and $\gamma-\mathrm{CD}$, respectively), derived from bacterial starch degradation. The shape of cyclodextrins is a truncated cone with a central cavity, due to the chair conformation of the glucopiranose units. The hydroxyl groups of glucopiranose units are oriented to the exterior of the molecule, giving it hydrophilic character. The central cavity of cyclodextrin is lined by skeletal carbons and ethereal oxygens of the glucose residues, which gives it a relatively lipophilic character. As a consequence of such structure, cyclodextrins are able to form inclusion complexes by encapsulating either partially or entirely a great number of molecules of suitable size into their central cavities. No covalent bonds are formed or broken during the inclusion complex formation and a fast equilibrium between the free and the complexed drug exists in an aqueous solution. The inclusion complex formation may favourably modify undesired biopharmaceutical properties of the drug, such as low chemical stability and limited aqueous solubility, leading to improved drug bioavailability. ${ }^{8-10}$

Cyclodextrins are biocompatible molecules with limited absorption in the gastrointestinal tract. Due to their high molecular weight and hydrophilic character,

\footnotetext{
$\dagger$ This article belongs to the Special Issue Chemistry of Living Systems devoted to the intersection of chemistry with life.

* Author to whom correspondence should be addressed. (E-mail: mariog@pharma.hr)
} 
cyclodextrins can permeate the gastrointestinal mucosa only with considerable difficulty. Therefore, cyclodextrins act as carriers of lipophilic drug molecules in the hydrophilic gastrointestinal media, delivering the drug to the surface of the gastrointestinal mucosa, where the complex dissociation and absorption of the free drug occur. ${ }^{9,11}$ Additionally, free cyclodextrins can interact with gastrointestinal mucosa, reversibly increasing its permeability for the drug, thus acting as permeation enhancers. ${ }^{7}$ The bulk of orally administered cyclodextrins is metabolised by the microflora present in the colon. The primary metabolites formed, such as maltodextrins, maltose and glucose are then absorbed into systemic circulation and finally metabolised to $\mathrm{CO}_{2}$ and $\mathrm{H}_{2} \mathrm{O}$. ${ }^{7}$

Among natural derivatives, $\beta C D$ is the most commonly used in pharmaceutical formulations due to a cavity size suitable for a wide range of drugs. ${ }^{8,9}$ However, due to the relatively strong hydrogen binding in the crystal lattice, the aqueous solubility of $\beta C D$ is much lower compared to the corresponding acyclic dextrins. The interaction of $\beta C D$ with lipophilic drugs may result in the inclusion complexes of limited aqueous solubility as well. Therefore, a series of chemically modified $\beta C D$ derivatives have been synthesized in order to extend the physicochemical properties and the inclusion capacity of the parent $\beta C D .{ }^{9}$ Several amorphous $\beta C D$ derivatives with improved aqueous solubilities have been developed, and some among them are proved to be safe even for parenteral application, such as hydroxypropyl-, sulphobutylether- or malthosyl- $\beta$-cyclodextrin., 11

The inclusion complex formation of ZAL with parent $\beta C D$ has already been reported, resulting in the significant improvement of its aqueous solubility. ${ }^{12}$ The effectiveness of cyclodextrins as drug carriers can be significantly influenced by the size of the cavity and the type of substituents present on the cyclodextrin core. In many cases, hydrophilic derivatives of $\beta C D$ have exhibited superior ability to solubilize and form complexes with different drugs compared to the parent $\beta \mathrm{CD} .{ }^{13-15}$ Therefore, it seemed of interest to extend the range of the investigated CDs to a series of native and modified cyclodextrins, in order to select the carriers with the greatest solubilizing and complexing efficacies towards ZAL. The interaction of ZAL with selected cyclodextrins in an aqueous solution was investigated by spectrofluorimetry and phase solubility studies, while ${ }^{1} \mathrm{H}-\mathrm{NMR}$ spectroscopy was used to confirm the actual inclusion complex formation and to give more insight into ZAL/CD binding mode.

\section{EXPERIMENTAL}

\section{Materials}

All water used was deionized and then twice distilled in an all-glass apparatus, first from an alkaline solution of
$\mathrm{KMnO}_{4}$. Zaleplon (99.7\% purity) was kindly donated by Belupo d. d. (Croatia). The cyclodextrins included in this study were natural, $\alpha-, \beta-, \gamma$-cyclodextrin $(\alpha \mathrm{CD}$, $\beta C D$ and $\gamma C D$, respectively) and $\beta$-cyclodextrin derivatives; hydroxypropyl- $\beta$-cyclodextrin (HP $\beta C D$ ) and randomly methylated- $\beta$-cyclodextrin (RAMEB), which were all obtained from Wacker Chemie GMBH (Germany). Average degrees of substitution per anhydroglucose unit are 0.9 and 1.8 for HP $\beta C D$ and RAMEB, respectively. Sulphobutylether- $\beta$-cyclodextrin sodium salt with a substitution degree of 0.9 (SBE- $\beta-\mathrm{CD})$ was obtained from CyDex Inc (USA). $\mathrm{D}_{2} \mathrm{O}$ (Sigma), $\mathrm{MeOH}$ (Sigma) and all other chemicals and solvents used in this study were of analytical reagent grade.

\section{Fluorescence studies}

Zaleplon stock solution was prepared by dissolving an appropriate amount of drug in a small amount of $\mathrm{MeOH}$ and then diluted with water to the final concentration of $0.1 \mathrm{mM}$. The final amount of $\mathrm{MeOH}$ in the stock solution was $1 \% v / v$. For each experiment, $3 \mathrm{~mL}$ of ZAL/CD solutions of appropriate concentration were prepared and left for an hour to equilibrate. The final concentration of zaleplon was $10 \mu \mathrm{M}$ and the cyclodextrin concentration varied from $0.1 \mathrm{mM}$ to $40 \mathrm{mM}$, depending on the cyclodextrin solubility. $\mathrm{MeOH}$ was $0.1 \% v / v$ in the final solutions. Fluorescence was measured by OLIS RSM 1000F spectrofluorimeter (Bogart, Georgia, USA) equipped with thermostatted cell at $25^{\circ} \mathrm{C}$. The excitation wavelength was $393 \mathrm{~nm}$ and the emission spectrum of pure zaleplon exhibited a maximum at $487 \mathrm{~nm}$. Each spectrum presented in titration curves is an average of 10000 fluorescence spectra measured in 10 seconds. Equilibrium constants were calculated by a global fit at all wavelengths performed with Specfit ${ }^{\mathbb{R}}$ software.

\section{Phase solubility studies}

Phase solubility studies of ZAL with different cyclodextrins were performed according to the method described by Higuchi and Connors. ${ }^{16}$ An excess amount of the drug $(50 \mathrm{mg}$ ) was added to $20 \mathrm{ml}$ of an aqueous cyclodextrin solution and stirred at a constant temperature $\left(25.0 \pm 0.5^{\circ} \mathrm{C}\right)$ in sealed containers for 72 hours until complexation equilibrium was reached. The concentrations of the cyclodextrins ranged from 0 to $48 \mathrm{mM}$, except for $\beta C D$, whose concentration ranged from 0 to $12.5 \mathrm{mM}$ due to its limited aqueous solubility. After the complexation equilibrium was reached, aliquots of the samples were filtered through $0.45 \mu \mathrm{m}$ Millipore membrane filter and the drug concentrations in the filtrate were determined spectrophotometrically at $335 \mathrm{~nm}$ (Ultrospec Plus, LKB, Pharmacia, Sweden). Preliminary studies showed that the presence of cyclodextrins did not interfere with ZAL absorbance at $335 \mathrm{~nm}$. 


\section{${ }^{1}$ H-NMR studies}

All NMR spectra were recorded at $300 \mathrm{~K}$ on a Bruker DRX 400 spectrometer (Karlsruhe, Germany) by using an inverse multinuclear (bbi) single-axis gradient $5 \mathrm{~mm}$ probe. The samples were prepared by dissolving approximately $0.5 \mathrm{mg}$ of the drug and $5 \mathrm{mg}$ of the cyclodextrin or an equimolar drug/cyclodextrin complex in $0.5 \mathrm{ml} \mathrm{D}_{2} \mathrm{O}$. The signal of residual water at $4.80 \mathrm{ppm}$ was used as an internal reference to avoid interferences. Two-dimensional diffusion ordered NMR spectroscopy (DOSY) was performed using the longitudinal eddy current delay experiment with bipolar gradients (ledbpgp $2 s$ standard program from Bruker pulse sequence library). The gradient intensity was varied linearly in 64 steps from 2 to $95 \%$ of the maximum gradient strength $\left(50 \mathrm{G} \mathrm{cm}^{-1}\right)$. The gradient pulse duration and diffusion delay were set at $4 \mathrm{~ms}$ and $50 \mathrm{~ms}$, respectively. $16 \mathrm{~K}$ data points were acquired in $f_{2}$, and 64 scans were collected for each gradient increment. The diffusion constants were determined by fitting an exponential decay function to the obtained peak intensities using the relaxation analysis module of Bruker TopSpin 2.5 program. Two-dimensional ROESY experiments were performed using the standard Bruker pulse program (roesytp) with a relaxation delay of $1.7 \mathrm{~s}$ and a mixing time of $300 \mathrm{~ms}$ under $\mathrm{cw}$ spinlock conditions. ROESY spectra were recorded with 2048 data points in $f_{2}, 400$ data points in $f_{1}$, and 64 scans were collected at each increment.

\section{RESULTS AND DISCUSSION}

In this paper we studied the interaction of natural and modified cyclodextrins with zaleplon in order to understand the behaviour of these complexes in an aqueous solution and to evaluate their potential for eventual use in medicine (Scheme 1).

Stability constants and the modalities of binding were our primal goals in this study. Zaleplon exhibits emission spectra in the visible region when excited at $393 \mathrm{~nm}$. This characteristic was used in study of the reactions with cyclodextrins which show no fluorescence in that region. Titration curves were measured

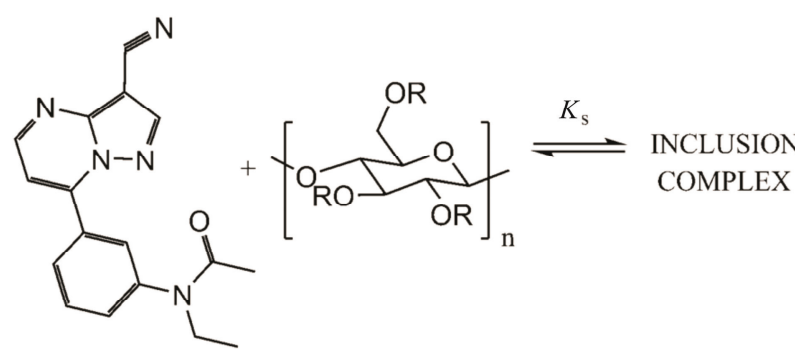

Scheme 1. Reaction of Zaleplon with Cyclodextrins

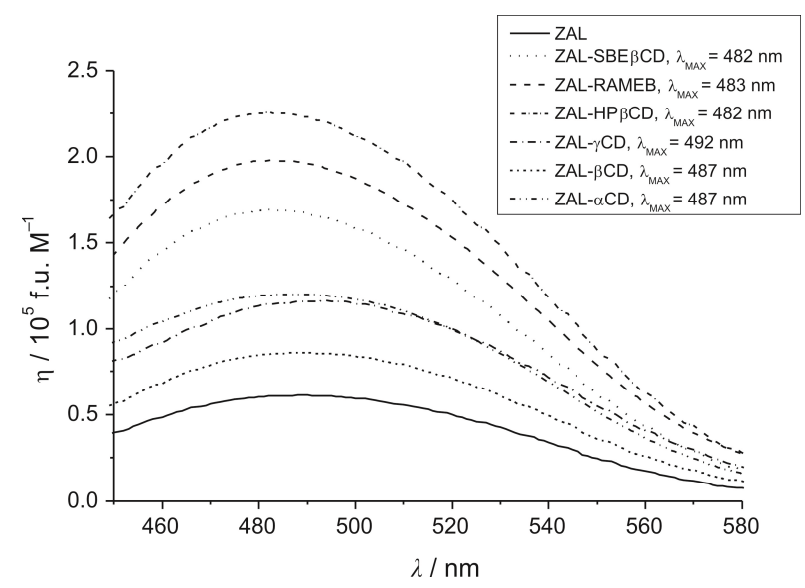

Figure 1. Spectral species obtained by spectrofluorimetric titrations of Zaleplon with natural and modified cyclodextrins. Molar fluorescence coefficients, $\eta$, are given in $10^{5}$ fluorescence units per mol dm${ }^{-3}$. Inset: Calculated spectral maxima of complexes.

with all cyclodextrins in order to establish the stoichiometry of the complex and the values of the stability constants. Figure 1 shows fluorescence spectra of zaleplon and its complexes with the investigated cyclodextrins. In all cases the intensity and the spectral maxima of the zaleplon emission spectra were changed upon addition of the $\mathrm{CD}$, indicating a change of polarity in the close proximity of the zaleplon molecule.

When a guest molecule is entrapped in the CD cavity, this microenvironment with a smaller polarity and a stronger rigidity would restrict the freedom of the guest molecule and consequently increase the fluorescence quantum yield. ${ }^{17,18}$ In addition, the steric hindrance of $\mathrm{CD}$ can protect the excited states from quenching processes and enhance the fluorescence efficiency. Titration curves were measured with all investigated cyclodextrins and a typical titration of ZAL with $\beta C D$ is shown in Figure 2. In all titrations an increase of quantum yield was observed during the titration (Figures S1-S5, supplemental).

All spectra in the titrations were analysed with Specfit ${ }^{19-21}$ software and only two spectrally active species were suggested by SVD (single value decomposition) statistical analysis, one attributed to zaleplon and the other one to its complex with cyclodextrin. This analysis suggested only 1 to 1 complex formation and did not indicate higher order complexes in all cases. Consequently, the proposed model is given by Eq. (1).

$$
\mathrm{ZAL}+\mathrm{CD} \rightleftarrows \mathrm{ZAL}-\mathrm{CD}, \quad K_{\mathrm{FL}}=\frac{[\mathrm{ZAL}-\mathrm{CD}]}{[\mathrm{ZAL}][\mathrm{CD}]}
$$

The stability constants $K_{\mathrm{FL}}$ for given model are summarized in Table 1. 


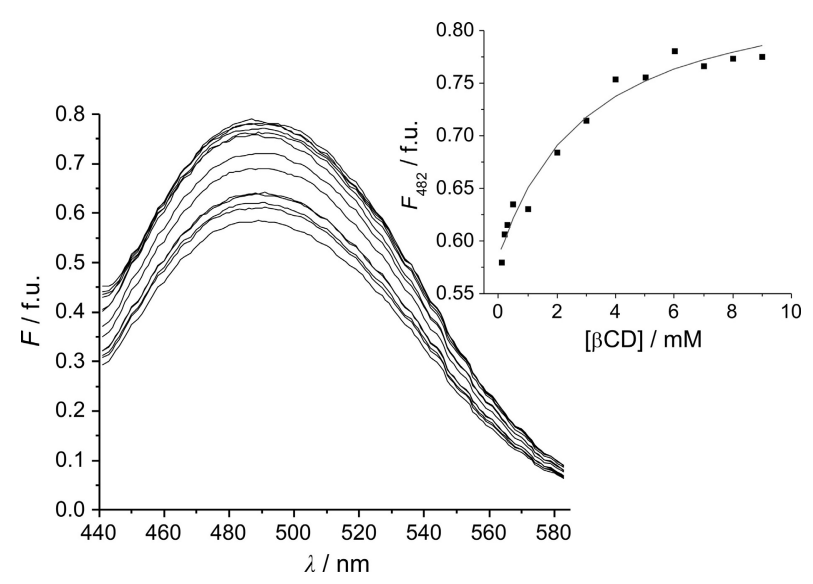

Figure 2. A typical titration of $10 \mu \mathrm{M}$ solution of ZAL with $\beta C D$ at $25^{\circ} \mathrm{C}, l=1 \mathrm{~cm}$. The concentration of $\beta C D$ was varied up to $9 \mathrm{mM}$. Fluorescence units (f.u.) are given as a ratio of signals obtained from sample and reference PMTs. Inset: Fitting curve at $482 \mathrm{~nm}$.

Stability constants obtained by spectrofluorimetric studies indicated that the ZAL/ $\beta C D$ complex has the highest stability of natural CDs, indicating the most appropriate size for fitting ZAL into its central cavity. The presence of the substituents on $\beta C D$ (RAMEB, SBE $\beta C D$ and $H P \beta C D$ ) probably sterically hinders the inclusion of the drug and consequently decreases the stability constants.

The solubilizing effect of each cyclodextrin derivative on the drug was investigated by phase solubility studies. In case of all cyclodextrins, ZAL solubility increased linearly $\left(r^{2}>0.98\right)$ as a function of CD concentration (Figure 3 ), showing an $A_{L}$ type of diagram, according to Higuchi and Connors. ${ }^{16}$

This suggested the formation of soluble inclusion complexes with 1:1 drug/cyclodextrin molar ratio, irrespective of the type of cyclodextrin used. This is in agreement with the results of spectrofluorimetric studies, which also indicated the same complex stoichiometry. The apparent stability constant of the inclusion complexes formed $\left(K_{\mathrm{SOL}}\right)$ was calculated according to the equation proposed by Higuchi and Connors ${ }^{16}$
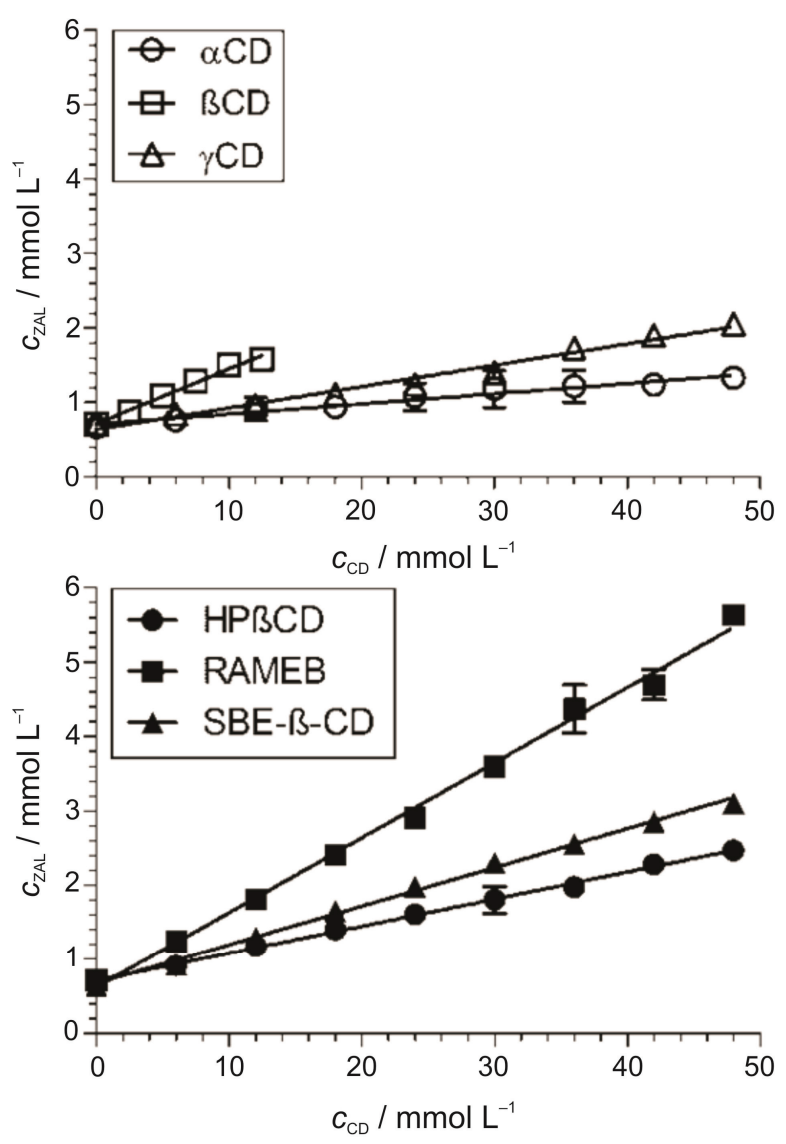

Figure 3. Phase solubility studies of ZAL with natural $(\alpha-, \beta$ and $\gamma-\mathrm{CD}$ ) and $\beta$-CD derivatives (HP $\beta C D$, RAMEB and $\mathrm{SBE} \beta \mathrm{CD})$ in water at $25^{\circ} \mathrm{C}($ mean $\pm \mathrm{SD})$.

$$
K_{\mathrm{SOL}}=\frac{\operatorname{tg} \alpha}{s_{0}(1-\operatorname{tg} \alpha)}
$$

where $\operatorname{tg} \alpha$ represents the slope of the phase solubility diagram and $s_{0}$ is the solubility of zaleplon in the absence of cyclodextrin. The obtained values of $K_{\mathrm{SOL}}$ are also presented in Table 1 . The slight discrepancy between $K_{\mathrm{s}}$ values obtained by spectrofluorimetric and phase solubility studies may be related to differences in experimental

Table 1. Stability constants of ZAL/CD complexes measured by fluorescence $\left(K_{\mathrm{FL}}\right)$ and solubility $\left(K_{\mathrm{SOL}}\right)$ studies and solubilization efficiency

\begin{tabular}{lrrc|}
\hline \multicolumn{1}{c}{$\mathrm{CD}$} & $K_{\mathrm{FL}} /\left(\mathrm{mol}^{-1} \mathrm{dm}^{3}\right)$ & $K_{\mathrm{SOL}}\left(\mathrm{mol}^{-1} \mathrm{dm}^{3}\right)$ & Solubilization efficiency \\
\hline$\alpha \mathrm{CD}$ & $141.25 \pm 1.70$ & $20.81 \pm 2.37$ & 1.99 \\
$\beta \mathrm{CD}$ & $331.13 \pm 1.17$ & $112.85 \pm 0.23$ & 2.24 \\
$\mathrm{HP} \beta C D$ & $16.98 \pm 1.23$ & $53.58 \pm 3.59$ & 3.48 \\
$\mathrm{RAMEB}$ & $107.15 \pm 1.28$ & $156.21 \pm 4.61$ & 7.83 \\
$\mathrm{SBE} \beta \mathrm{CD}$ & $67.61 \pm 1.07$ & $84.83 \pm 0.44$ & 4.80 \\
$\gamma \mathrm{CD}$ & $9.33 \pm 1.34$ & $41.10 \pm 3.03$ & 2.85 \\
\hline
\end{tabular}


(1)

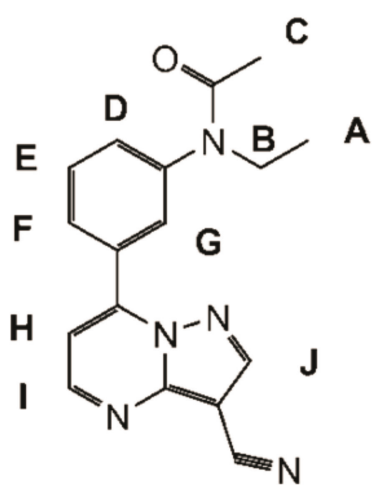

(2)

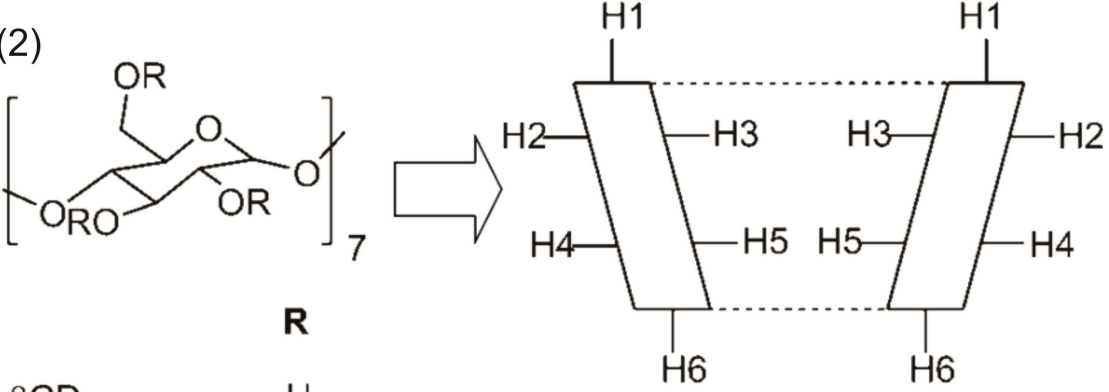

RAMEB $\quad \mathrm{CH}_{3}$

Figure 4. Structures and proton labelling of zaleplon (1) and the cyclodextrins used in the study (2).

techniques, particularly in the drug concentration and presence of co-solvents. In the spectrofluorimetric study, the drug concentration was kept constant and a small amount $(0.1 \% v / v)$ of methanol as co-solvent was used. On the contrary, the phase solubility studies were performed in pure aqueous solutions of the cyclodextrin where the drug concentration varied as a function of cyclodextrin amount in the sample. Furthermore, the stability constant determined from the phase solubility studies frequently corresponds to several different solubilisation phenomena, such as inclusion and non-inclusion complex formation, aggregation etc. that exist simultaneously in non-ideal aqueous solutions of cyclodextrins. All this may contribute to differences of stability constants measured by both methods, as previously reported by Loftsson et al. ${ }^{22}$

Both spectrofluorimetric and phase solubility studies showed that among natural cyclodextrins, $\beta C D$ formed the most stabile inclusion complexes with ZAL, while the stability of ZAL complexes with $\alpha \mathrm{CD}$ and $\gamma \mathrm{CD}$ was significantly lower. This can probably be attributed to the differences of central cavity sizes between natural CDs. The central cavity of $\alpha \mathrm{CD}$ is probably too small to suitably fit the guest, resulting in a lower complexing affinity towards ZAL than in the case of $\beta C D$. On the other hand, the excessively large cavity of $\gamma \mathrm{CD}$ did not allow sufficiently strong interaction between the host and the guest molecule. Similar findings were also observed by other authors. ${ }^{23,24}$

To estimate the solubilizing efficiency of each cyclodextrin, the ratio between ZAL solubility in the most concentrated cyclodextrin solution and in pure water was calculated (Table 1). The solubilizing efficiency of natural CDs followed the trend $\gamma \mathrm{CD}>\beta C D>\alpha C D$. Although $\beta C D$ formed the most stabile complexes, its solubilizing efficiency was somewhat reduced due to its limited aqueous solubility. ${ }^{25}$ All $\beta C D$ derivatives showed superior solubilizing efficiency compared to the parent $\beta C D$ (Table 1) and RAMEB was found to be the most effective complexing agent for ZAL. The substitution of the hydroxyl group at the $\beta C D$ core with methyl groups resulted in the remarkable increase of the aqueous solubility of the formed carrier. ${ }^{11}$ This probably led to a higher solubilisation efficiency of RAMEB for ZAL, compared to the parent $\beta C D$. The surface activity of the methylated cyclodextrins probably additionally contributed to the overall solubilizing effect. ${ }^{26}$ Furthermore, the presence of the rather lipophilic methoxy groups at the $\beta C D$ core might allow a stronger interaction with the lipophilic guest molecule, stabilizing its inclusion into the host cavity (Table 1).

In the case of other $\beta C D$ derivatives, a decrease of solubilizing efficiency and complex stability was observed. The introduction of more bulky substituents on the $\beta C D$ core, such as the hydroxypropylated and sulphobutylated groups, lowered the complexation affinity of such derivatives for ZAL by providing a hydrogen bonding source for the water molecules, which in turn decreased the energy difference between the water molecules included into the CD central cavity and those in the bulk of the solution. Furthermore, the presence of bulky, highly hydrated or highly charged groups near the $\beta C D$ cavity may inhibit the approach of a hydrophobic molecule, ${ }^{9}$ thus decreasing the affinity of such derivatives for complexation with ZAL (Table 1).

The increased aqueous solubility of the drug and enhancement of its fluorescence in the presence of cyclodextrins observed by the phase solubility studies and spectrofluorimetry cannot be taken as an ultimate proof of the actual formation of an inclusion complex. These techniques cannot provide a clear answer about the drug/cyclodextrin interaction mode (inclusion or adsorption), or provide an insight into the structure of the formed complexes. ${ }^{27}$ Therefore, the interaction between ZAL and $\beta C D$ or RAMEB was further characterised by ${ }^{1} \mathrm{H}-\mathrm{NMR}$ studies. The assignment of ${ }^{1} \mathrm{H}$ chemical shifts of ZAL, $\beta C D$ and RAMEB was done according to the related literature ${ }^{27-29}$ and corroborated by COSY spectroscopy (data not shown). The proton labels of ZAL and the studied cyclodextrins are presented in Figure 4. 
Table 2. ${ }^{1} \mathrm{H}-\mathrm{NMR}$ chemical shifts of $\beta$-CD and RAMEB in free and complex form, determined in $\mathrm{D}_{2} \mathrm{O}$ at $300 \mathrm{~K}$. The multiplicities of peaks are indicated in brackets (s-singlet; d-doublet; dd-doublet of doublets; t-triplet)

\begin{tabular}{|c|c|c|c|c|c|c|}
\hline \multirow{2}{*}{$\begin{array}{l}\text { proton } \\
\text { label }\end{array}$} & \multirow{2}{*}{$\frac{\beta C D}{\delta / \mathrm{ppm}}$} & \multicolumn{2}{|c|}{$\beta C D$ complex } & \multirow{2}{*}{$\frac{\text { RAMEB }}{\delta / \mathrm{ppm}}$} & \multicolumn{2}{|c|}{ RAMEB complex } \\
\hline & & $\delta / \mathrm{ppm}$ & $\Delta \delta^{(\mathrm{a})} / \mathrm{ppm}$ & & $\delta / \mathrm{ppm}$ & $\Delta \delta^{(\mathrm{a})} / \mathrm{ppm}$ \\
\hline H1 & $5.165(d)$ & $5.159(\mathrm{~d})$ & -0.006 & $5.366(d)$ & $5.360(d)$ & -0.006 \\
\hline $\mathrm{H} 2$ & $3.757(\mathrm{dd})$ & $3.753(\mathrm{dd})$ & -0.004 & - & - & - \\
\hline $\mathrm{H} 3$ & $4.055(\mathrm{t})$ & $4.027(\mathrm{t})$ & -0.028 & - & - & - \\
\hline $\mathrm{H} 4$ & $3.674(\mathrm{t})$ & $3.668(\mathrm{t})$ & -0.005 & - & - & - \\
\hline H5 & $3.943(\mathrm{~s})$ & $3.927(\mathrm{~s})$ & -0.017 & - & - & - \\
\hline H6 & $3.968(\mathrm{~s})$ & $3.956(\mathrm{~s})$ & -0.012 & - & - & - \\
\hline Me 2' & - & - & - & $3.662(\mathrm{~s})$ & $3.662(\mathrm{~s})$ & 0.000 \\
\hline Me $6^{\prime}$ & - & - & - & $3.490(\mathrm{~s})$ & $3.485(\mathrm{~s})$ & -0.005 \\
\hline
\end{tabular}

(a) $\Delta \delta=\delta_{\text {complex }}-\delta_{\text {free }}$

In ${ }^{1} \mathrm{H}-\mathrm{NMR}$ spectra of all studied binary systems, the drug signals were well separated from those of cyclodextrins, with the exception of the $\mathrm{H}_{\mathrm{b}}$ signal of ZAL, which was overlapped by the signals of cyclodextrin protons. No new peaks were observed in ${ }^{1} \mathrm{H}-\mathrm{NMR}$ spectra of ZAL/CDs samples, indicating that the guest molecule was in a rapid exchange between free and complex form, relative to the NMR timescale. ${ }^{30} \beta C D$ exhibited a well resolved ${ }^{1} \mathrm{H}-\mathrm{NMR}$ spectrum and its assignments are presented in Table 2.

The H3 and H5 protons, which are lining the interior of the cyclodextrin central cavity, as well as the H6 proton, which is located at the narrower end of the cyclodextrin torus, experienced a shielding effect resulting in their up-field shift $(\Delta \delta<0)$. Other $\beta C D$ protons, namely $\mathrm{H} 1$, $\mathrm{H} 2$ and $\mathrm{H} 4$, which are located on the exterior of the cyclodextrin molecule showed only a minor change of their resonance. This may be taken as a clear suggestion of an inclusion complex formation between ZAL and $\beta C D$. In particular, the observed shielding effect can be attributed to the diamagnetic anisotropy effect due to the inclusion of a group rich in $\pi$-electrons into the hydrophobic central cavity of the cyclodextrin molecule. ${ }^{31}$ Furthermore, the fact that the shielding effect was observed for $\mathrm{H} 3, \mathrm{H} 5$ and H6 indicated that the ZAL molecule penetrated deeply into the cyclodextrin central cavity. The higher shielding effect on the $\mathrm{H} 3$ proton with respect to those of $\mathrm{H} 5$ and $\mathrm{H} 6$ $(\Delta \delta \mathrm{H} 3>\Delta \delta \mathrm{H} 5 \geq \Delta \delta \mathrm{H} 6)$, shows that the inclusion of ZAL into $\beta C D$ cavity probably occurred via a more accessible wider side of the cyclodextrin molecule (i.e. the secondary hydroxyl rim of the torus) ${ }^{13,27}$

Further information about ZAL/ $\beta C D$ inclusion complex formation was obtained by analysing the chemical shift values of the drug molecule (Table 3 ).

Table 3. ${ }^{1} \mathrm{H}-\mathrm{NMR}$ chemical shifts of ZAL in free and complex form with $\beta$-CD and RAMEB determined in $\mathrm{D}_{2} \mathrm{O}$ at $300 \mathrm{~K}$. The multiplicities of peaks are indicated in brackets (s-singlet; d-doublet; t-triplet; q-quartet)

\begin{tabular}{lccccc}
\hline & ZAL & \multicolumn{2}{c}{$\beta$ CD complex } & \multicolumn{2}{c}{ RAMEB complex } \\
\hline proton label & $\delta / \mathrm{ppm}$ & $\delta / \mathrm{ppm}$ & $\Delta \delta^{(\mathrm{a})} / \mathrm{ppm}$ & $\delta / \mathrm{ppm}$ & $\Delta \delta^{(\mathrm{a})} / \mathrm{ppm}$ \\
\hline $\mathrm{Ha}$ & $1.224(\mathrm{t})$ & $1.294(\mathrm{t})$ & 0.069 & $1.293(\mathrm{t})$ & 0.069 \\
$\mathrm{Hb}$ & $3.859(\mathrm{q})$ & - (b) & - & - (b) & - (b) \\
$\mathrm{Hc}$ & $2.012(\mathrm{~s})$ & $2.063(\mathrm{~s})$ & 0.051 & $2.043(\mathrm{~s})$ & 0.031 \\
$\mathrm{Hd}$ & $8.089(\mathrm{~d})$ & $8.221(\mathrm{~d})$ & 0.132 & $8.264(\mathrm{~d})$ & 0.175 \\
$\mathrm{He}$ & $7.856(\mathrm{t})$ & $7.979(\mathrm{t})$ & 0.123 & $7.990(\mathrm{t})$ & 0.134 \\
$\mathrm{Hf}$ & $7.544(\mathrm{~d})$ & $7.651(\mathrm{~d})$ & 0.108 & $7.650(\mathrm{~d})$ & 0.106 \\
$\mathrm{Hg}$ & $8.028(\mathrm{~s})$ & $8.067(\mathrm{~s})$ & 0.039 & $8.094(\mathrm{~s})$ & 0.066 \\
$\mathrm{Hh}$ & $7.728(\mathrm{~d})$ & $7.670(\mathrm{~d})$ & -0.058 & $7.716(\mathrm{~d})$ & -0.012 \\
$\mathrm{Hi}$ & $8.945(\mathrm{~d})$ & $8.965(\mathrm{~d})$ & 0.020 & $9.063(\mathrm{~d})$ & 0.118 \\
$\mathrm{Hj}$ & $8.690(\mathrm{~s})$ & $8.722(\mathrm{~s})$ & 0.033 & $8.701(\mathrm{~s})$ & 0.011 \\
\hline
\end{tabular}

(a) $\Delta \delta=\delta_{\text {complex }}-\delta_{\text {free. }}$

(b) overlapped by cyclodextrin signal. 
The majority of the drug protons showed a down-field shift in the presence of $\beta \mathrm{CD}(\Delta \delta>0)$, while only $\mathrm{Hh}$ proton was shifted up-field. A down-field shift may be related to changes in the local polarity due to the inclusion of the drug into the lipophilic central cavity of the cyclodextrin molecule or to the deshielding effects caused by the van der Waals interaction between the drug and the carbohydrate chains. ${ }^{31}$ The most affected ZAL protons were $\mathrm{Hd}, \mathrm{He}$ and $\mathrm{Hf}$, indicating that the interaction of the phenyl ring of the drug molecule with the central cavity of $\beta C D$ was the most intense one. Furthermore, the $\mathrm{Ha}$ and $\mathrm{Hc}$ protons experienced a somewhat stronger shielding effect compared to Hi and $\mathrm{Hj}$, which may indicate that $\mathrm{Ha}$ and $\mathrm{Hc}$ are located more deeply into lipophilic central cavity of $\beta C D$. Up-field shift of Hh proton indicated that this proton is close to a host atom rich with $\pi$-electrons, which in this case is associated with the oxygen atom of $\beta C D$, but also reflected some conformational changes produced by the inclusion. ${ }^{32}$ Based on these results, it may be assumed that the whole drug molecule is included into the central cavity of the $\beta C D$.

${ }^{1} \mathrm{H}-\mathrm{NMR}$ spectrum of RAMEB was not well resolved because it is not a single pure compound, but rather a complex mixture of randomly methylated molecules of $\beta C D .^{29}$ As a result, only some of the RAMEB signals could be unambiguously identified (Table 2). While comparing the chemical shifts of $\mathrm{H} 1, \mathrm{Me} 2$ and Me6 of RAMEB in free and complex form (Table 2), it may be seen that these protons experienced only minor shielding effect or none at all. Signals of other RAMEB protons are uncertain and cannot be used for interpretation of the drug inclusion mode. Therefore, information about the inclusion complex formation between ZAL and RAMEB was deduced solely on the basis of chemical shift changes of the drug (Table 3). Similarly as in the case of $\beta \mathrm{CD}, \mathrm{Hd}, \mathrm{He}$ and $\mathrm{Hf}$ were the most influenced protons of the drug, indicating a similar guest/host binding mode as well. Interestingly, the chemical shift change of those protons was more pronounced with RAMEB than in the case of $\beta C D$. This increasing chemical shift perturbation can be attributed to a higher fraction of bound drug molecules, which is consistent with the stability constant values obtained by phase solubility studies. We have previously observed similar behaviour in the case of the inclusion complexes between bupivacaine hydrochloride and several $\beta C D$ derivatives. ${ }^{33}$ Furthermore, the more pronounced downfield shift of Hi proton and the reduced up-field shift of Hh proton observed for RAMEB complex (Table 3) may be attributed to a changed microenvironment due to the presence of the methyl groups on the $\beta C D$ core.

Further evidence for the inclusion complex formation between ZAL and the selected cyclodextrin derivatives was obtained by DOSY spectroscopy. The average
Table 4. The average diffusion coefficients of drug and both cyclodextrins in free or in the complex form. The values in brackets represent the standard deviation

\begin{tabular}{lcc}
\hline & \multicolumn{2}{c}{$D \times 10^{10} /\left(\mathrm{m}^{2} \mathrm{~s}^{-1}\right)$} \\
\cline { 2 - 3 } & $\mathbf{Z A L}_{\text {average }}$ & $\mathbf{C D}_{\text {average }}$ \\
\hline ZAL & $5.196(0.013)$ & - \\
$\beta C D$ & - & $3.103(0.014)$ \\
$\beta C D-c o m p l e x$ & $3.526(0.041)$ & $3.107(0.014)$ \\
RAMEB & - & $2.428(0.080)$ \\
RAMEB-complex & $2.460(0.008)$ & $2.380(0.001)$ \\
\hline
\end{tabular}

diffusion coefficient of the drug was determined analysing the DOSY signals of $\mathrm{Ha}, \mathrm{Hc}, \mathrm{Hf}, \mathrm{Hd}, \mathrm{Hi}$ and $\mathrm{Hj}$ protons. For $\beta C D$, the average diffusion coefficient was determined analysing the DOSY signals of $\mathrm{H} 1, \mathrm{H} 2, \mathrm{H} 3$, $\mathrm{H} 4$ and H6, while in case of RAMEB, H1, Me2 and Me6 DOSY signals were taken into calculation. A pair of values was obtained for both cyclodextrin complexes, because the average diffusion coefficients were calculated by taking into account the DOSY signals of the drug and the signals of the corresponding cyclodextrin. The obtained results are presented in Table 4.

In the presence of both cyclodextrins (Table 4), ZAL showed a significant reduction of the diffusion rate, compared to that of the pure drug. Since the viscosity change is negligible in the diluted cyclodextrin solution compared to pure water, this change confirmed the formation of a relatively stable inclusion complexes of ZAL with both cyclodextrins studied. The extent of the reduction of the diffusion coefficient is well correlated with the molecular weight of cyclodextrins. In the case of $\beta C D$ (MW $1135 \mathrm{~g} \mathrm{~mol}^{-1}$ ), the drug diffusion rate was reduced approximately $32 \%$, while in the case of RAMEB (MW $1303 \mathrm{~g} \mathrm{~mol}^{-1}$ ) the reduction was about 50 $\%$, both compared to the diffusion coefficient of the pure drug. This is in agreement with the molecular weight differences of complexes formed. For both complexes the diffusion coefficients calculated from DOSY signals of ZAL and CDs are slightly different. In the presence of both cyclodextrins, the diffusion of the drug was significantly reduced with respect to that of the free drug, although it is not completely the same as that calculated on the basis of DOSY signals of the CDs (Table 4). This may be related to the fast equilibrium between the bonded and the free drug, as suggested by Pescitelli et al. ${ }^{34}$ Assuming that the bound and the free ZAL undergo a fast exchange on the diffusion timescale, the observed ZAL diffusion coefficient $\left(D_{\text {obs }}\right)$ is an average of the bound $\left(D_{\text {complex }}\right)$ and the free $\left(D_{\text {free }}\right)$ drug:

$$
D_{\text {obs }}=f_{\text {free }} \times D_{\text {free }}+f_{\text {complex }} \times D_{\text {complex }}
$$



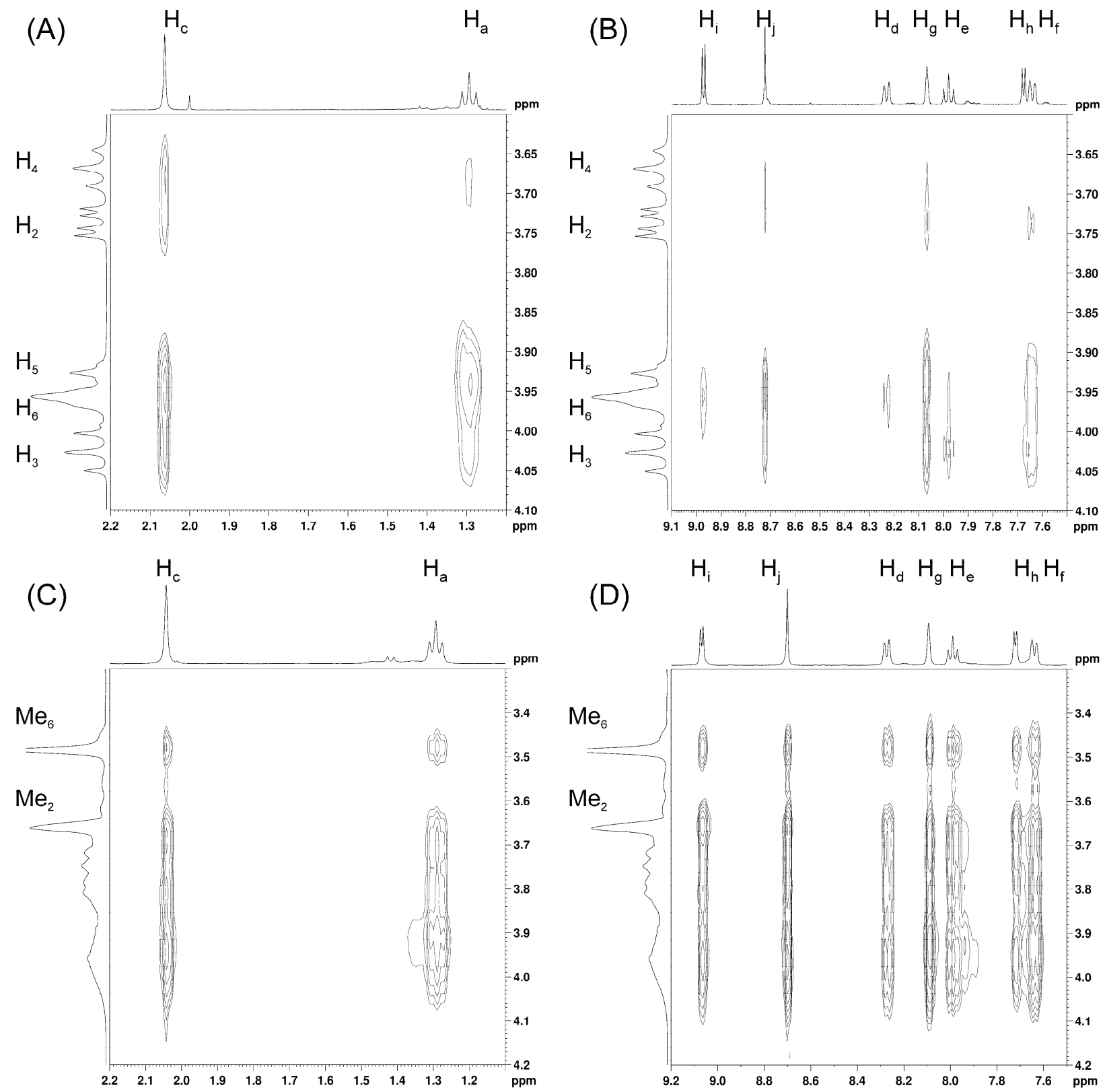

Figure 5. Partial contour plots of $2 \mathrm{D}$ ROESY spectrum of ZAL in the presence of $\beta C D(A, B)$ and RAMEB $(C, D)$ in $D_{2} O$ at 300 K. For the proton labelling see Figure 4.

where $f_{\text {free }}$ and $f_{\text {complex }}$ are the fractions of the free ZAL and the drug bonded to cyclodextrin complex. The fraction of the drug in complex may be expressed as:

$$
f_{\text {complexed }}=1-f_{\text {free }}
$$

Substituting Eq. (4) into Eq. (3) gives:

$$
f_{\text {complexed }}=\frac{\left(D_{\text {free }}-D_{\text {obs }}\right)}{\left(D_{\text {free }}-D_{\text {complexed }}\right)}
$$

Recognising that molecular weight of ZAL (305.34 $\mathrm{g} \mathrm{mol}^{-1}$ ) is much smaller than that of cyclodextrins, it can be assumed that the diffusion coefficient of the host is not greatly perturbed by the binding of a small guest molecule. Therefore, the diffusion coefficient of the host-guest complex can be assumed to be the same as that of the non-complexed host molecule alone. ${ }^{35-37}$ Consequently, the Eq. (5) may be transformed to:

$$
f_{\text {complexed }}=\frac{\left(D_{\text {free }}-D_{\text {obs }}\right)}{\left(D_{\text {free }}-D_{\mathrm{CD}}\right)}
$$


Using the Eq. (6), the fraction of ZAL in the complex with $\beta C D$ and RAMEB was calculated to be $88.87 \%$ and $99.79 \%$, respectively. This confirmed the phase solubility data, which indicated RAMEB as a better complexing and solubilizing agent for ZAL compared to the parent $\beta C D$.

Two-dimensional rotating-frame Overhauser effect spectroscopy (ROESY) is a powerful technique for investigation of the inter- and intra-molecular interactions. The presence of NOE cross-peak between the protons of two different species in a 2D ROESY spectrum is an indication that they are in a spatial contact within $3-5 \AA \AA^{38}$ Therefore, to further confirm the actual inclusion complex formation and to give more insight into the binding mode of ZAL and the selected cyclodextrins, 2D ROESY experiments were performed. In the 2D ROESY spectrum of ZAL/ $\beta C D$ complex, two groups of intermolecular NOE cross-peaks were observed. The first group belongs to the interaction of the $\mathrm{Ha}$ and $\mathrm{Hc}$ protons of ZAL with the $\beta \mathrm{CD}$ protons (Figure $5 \mathrm{~A}$ ), while the second group corresponds to the interaction of the aromatic $\mathrm{ZAL}$ protons $(\mathrm{Hd}-\mathrm{HHj})$ and the $\beta C D$ protons (Figure $5 \mathrm{~B}$ ).

Both methyl protons ( $\mathrm{Ha}$ and $\mathrm{Hc}$ ) of the $\mathrm{N}$ ethylacetamide group of ZAL showed strong NOE cross-peaks with the $\mathrm{H} 3, \mathrm{H} 6$ and $\mathrm{H} 5$ protons of the $\beta C D$, confirming that they are included deeply into the cyclodextrin central cavity. The free rotation of $N$ ethylacetamide group might allow its simultaneous interaction with both internal protons of the $\beta \mathrm{CD}$. At the same time, NOE cross-peaks of the $\mathrm{Ha}$ and $\mathrm{Hc}$ protons of ZAL with the external $\beta C D$ protons were also observed, but their intensity was significantly lower compared to those of the internal protons (Figure 5A), indicating that the inclusion of the $\mathrm{N}$ ethylacetamide into the central cavity was the dominant binding mode. Interestingly, all aromatic protons showed NOE cross-peaks which indicated their interactions with both the internal ( $\mathrm{H} 3$ and $\mathrm{H} 5)$ but also the H6 protons, except in the case of Hi and Hd protons. Those protons did not show any NOE crosspeaks corresponding to the interaction with the $\mathrm{H} 3$ proton of the $\beta C D$ (Figure 5B). Such results may lead to the conclusion that in the case of ZAL/BCD complex, two binding modes exist simultaneously in the solution: the one in which the binding occurs by the inclusion of the phenyl ring of ZAL into the $\beta C D$ central cavity via the wider rim of the cyclodextrin cone (A) and the other by the inclusion of the pyrazolo[1,5a]pyrimidine ring (B), as suggested in Figure 6. This structure would allow the interaction of the methyl protons of the $N$-ethylacetamide group of ZAL with the external protons of the adjacent $\beta C D$ molecule, resulting in weak NOE cross-peaks which may be observed in Figure 5A. But taking into account the
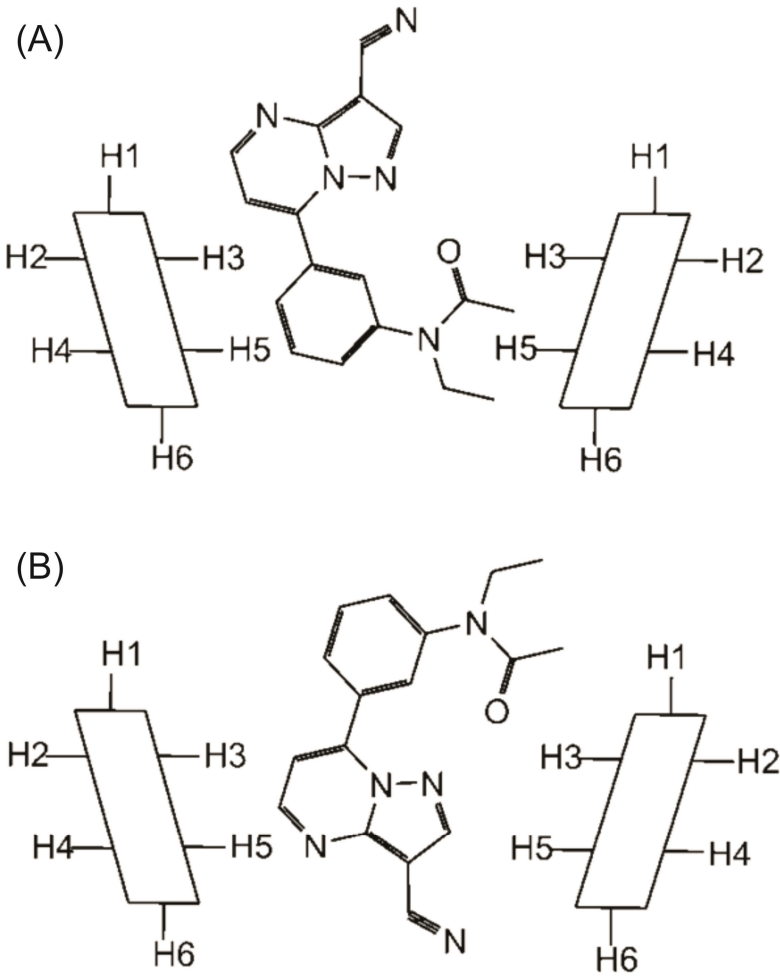

Figure 6. The proposed structures of ZAL/ $\beta C D$ inclusion complex deduced on the basis of ${ }^{1} \mathrm{H}-\mathrm{NMR}$ spectroscopy results.

stronger shielding effect for the Hd-Hf protons observed by the one-dimensional ${ }^{1} \mathrm{H}-\mathrm{NMR}$ spectroscopy (Table 2), it might be concluded that the type A of inclusion (Figure 6A) is probably the dominant binding mode. The inclusion of one ZAL into two $\beta C D$ molecules can be excluded, because phase solubility studies and spectrofluorimetry did not give any indication of a higher-order complex formation. Furthermore, the formation of higher order complexes is not very likely for drugs whose geometry is similar to ZAL due to sterical reasons, as discussed recently by Messner et al. ${ }^{39}$

ROESY spectra of ZAL/RAMEB complex also showed two sets of NOE cross-peaks: the one belonging to the interaction of the $\mathrm{Ha}$ and $\mathrm{Hc}$ protons of ZAL with the RAMEB protons (Figure 5C) and the other belonging to the interaction of the aromatic protons $(\mathrm{Hd}-\mathrm{Hj})$ of ZAL with the protons of the cyclodextrin derivative (Figure 5D). All signals were very intense and their overlapping was observed. Although these results unquestionably confirmed the inclusion complex formation between ZAL and RAMEB, the elucidation of its structure might be rather ambiguous. However, the same binding mode as in the case of $\beta C D$ can probably be assumed. The strong NOE cross-peaks between all the drug protons and the Me2 and Me6 of RAMEB confirmed their strong interaction with the drug molecule upon the inclusion complex formation. 


\section{CONCLUSION}

The results of the spectrofluorimetric and phase solubility studies showed that the parent $\beta C D$ and RAMEB are the most appropriate complexing agents for ZAL among the different studied cyclodextrin derivatives. The inclusion complex formation caused an enhanced florescence quantum yield and significantly improved the aqueous solubility of the drug. One- and twodimensional ${ }^{1} \mathrm{H}-\mathrm{NMR}$ studies confirmed the actual inclusion complex formation and gave some insight into the drug/cyclodextrin binding mode. Based on ROESY spectra, it was concluded that two binding modes of the ZAL/ $\beta C D$ complex exist simultaneously in the solution. The first one occurs by the inclusion of the phenyl ring of ZAL into the $\beta C D$ central cavity via the wider rim of the cyclodextrin cone and the second occurs by the inclusion of the pyrazolo[1,5-a] pyrimidine ring of ZAL. The first one seems to be the dominant $\mathrm{ZAL} / \mathrm{BCD}$ binding mode.

Supplementary Materials. - Supporting informations to the paper are enclosed to the electronic version of the article. These data can be found on the website of Croatica Chemica Acta (http://public.carnet.hr/ccacaa).

Acknowledgements. This work was supported by grants 0060061247-0978 and 006-0061117-1244 of the Ministry of Science, Education and Sports of the Republic of Croatia. Financial support by the Access to Research Infrastructures activity in the 7th Framework Programme of the EC (Contract 228461, EAST-NMR) for conducting the research is gratefully acknowledged. The authors are thankful to Prof. Katalin E. Kövér and Prof. Istvan Banyai for their help in the conducting of ${ }^{1} \mathrm{H}-\mathrm{NMR}$ experiments and their fruitful comments on the results.

\section{REFERENCES}

1. K. T. Bain, Am. J. Geriatr. Pharmacother. 4 (2006) 168-192.

2. S. H. Tariq and S. Pulisetty, Clin. Geriatr. Med. 24 (2008) 93-105.

3. W. E. Heydorn, Exp. Opin. Invest. Drugs 9 (2000) 841-858.

4. A. Waghmare, Y. Pore, and B. Kuchekar, AAPS Pharm. Sci. Tech. 9 (2008) 536-543.

5. M. E. Brewster and T. Loftsson., Adv. Drug Del. Rev. 59 (2007) 645-666.

6. A. S. Rosen, P. Fournie, M. Darwish, P. Danjou, and S. M. Troy, Biopharm. Drug Dispos. 20 (1999) 171-175.

7. T. Loftsson, S. B. Vogensen, M. E. Brewster, and F. Konráđsdóttir, J. Pharm. Sci. 96 (2007) 2532-2546.

8. E. M. M. Del Valle, Process Biochem. 39 (2004) 1033-1046.

9. R. Challa, A. Ahuja, and R. K. Khar, AAPS Pharm. Sci. Tech. 6 (2005) 329-357.

10. R. L. Carrier, L. A. Miller, and I. Ahmed, J. Control. Release 123 (2007) 78-99.
11. T. Loftsson and D. Duchene, Int. J. Pharm. 329 (2007) 1-11.

12. D. Doiphode, S. Gaikwad, Y. Pore, B. Kuchekar, and S. Late, J. Incl. Phenom. Macrocycl. Chem. 62 (2008) 43-50.

13. M. Jug and M. Bećirević-Laćan, Drug Dev. Ind. Pharm. 30 (2004) 1051-1060.

14. M. Cirri, F. Maestrelli, S. Orlandini, S. Furlanetto, S. Pinzauti, and P. Mura, J. Pharm. Biomed. Anal. 37 (2005) 995-1002.

15. S. M. Badr-Eldin, S. A. Elkheshen, and M. M. Ghorab, Eur. J. Pharm. Biopharm. 70 (2008) 819-827.

16. T. Higuchi and K. A. Connors, Adv. Anal. Chem. Instrum. 4 (1965) 117-212.

17. G. Zhang, S. Shuang, C. Dong, and J. Pan, Spectrochim. Acta A Mol. Biomol. Spectrosc. 59 (2003) 2935-2941.

18. M. Shamsipur, A. Yari, and H. Sharghi, Spectrochim. Acta A Mol. Biomol. Spectrosc. 62 (2005) 372-376.

19. H. Gampp, M. Maeder, and C. J. Meyer, A.D. Zuberbühler, Talanta 33 (1986) 943-101.

20. H. Gampp, M. Maeder, and C. J. Meyer, A.D. Zuberbühler, Talanta 32 (1985) 95-101.

21. H. Gampp, M. Maeder, and C. J. Meyer, A.D. Zuberbühler, Talanta 32 (1985) 251-264.

22. T. Loftsson, M. Masson, and M. E. Brewster, J. Pharm. Sci. 93 (2004) 1091-1099.

23. M. T. Faucci, F. Melani, and P. Mura, Chem. Phys. Let. 358 (2002) 383-390.

24. M. D. Veiga, M. Merino, M. Cirri, F. Maestrelli, and P. Mura, J. Incl. Phen. Macrocyc. Chem. 53 (2005) 77-83.

25. K. H. Frömming and J. Szejtli, Cyclodextrins in Pharmacy, 1994, Kluwer Academic Publishers: Dordrecht, Boston, London, p. 10.

26. F. Csempesz, A. Süle, and I. Pusks, Colloids Surf. 354 (2010) $308-310$.

27. F. J. B. Veiga, C. M. Fernandes, R. A. Carvalho, and C. F. G. C. Geraldes, Chem. Pharm. Bull. 49 (2001) 1251-1256.

28. Ch. Bharathi, K. J. Prabahar, Ch. S. Prasad, M. S. Kumar, S. Magesh, V. K. Handa, R. Dandala, and A. Naidu., J. Pharm. Biomed. Anal. 44 (2007) 101-109.

29. A. Hazekamp and R. Verpoorte., Eur. J. Pharm. Sci. 29 (2006) 340-347.

30. T. D. Thi, K. Nauwelaeters, M. Froeyen, L. Baudemprez, M. Van Speybroeck, P. Augustijns, P. Annaert, J. Martens, J. Van Humbeeck, and G. Van den Mooter, J. Pharm. Sci. 99 (2010) 3863-3873.

31. F. Djedaine, S. Lin, B. Perly, and D. Wouessidjewe, J. Pharm. Sci. 79 (1990) 643-646.

32. L. Ribeiro, R. A. Carvalho, D. C. Ferreira, and F. J. B. Veiga, Eur. J. Pharm. Sci. 24 (2005) 1-13.

33. M. Jug, N. Mennini, F. Melani, F. Maestrelli, and P. Mura, Chem. Phys. Lett. 500 (2010) 347-354.

34. G. Pescitelli, A. R. Bilia, M. C. Bergonzi, F. F. Vincieri, and L. D. Bari, J. Pharm. Biomed. Anal. 52 (2010) 479-483.

35. C. Jullian, S. Miranda, G. Zapata-Torres, F. Mendizabal, and C. Olea-Azar, Bioorg. Med. Chem. 15 (2007) 3217.

36. R. Grillo, N. F. S. Melo, C. M. Moraes, A. H. Rosa, J. A. F. Roveda, C. M. S. Menezes, E. I. Fereira, and L. F. Franceto, J. Biol. Phys. 33 (2007) 445-453.

37. A. Calderini and F. B. T. Pessine, J. Incl. Phenom. Macrocycl. Chem. 60 (2008) 369-377.

38. L. Ribeiro, R. A. Carvalho, D. C. Ferreira, and F. J. B. Veiga, Eur. J. Pharm. Sci. 24 (2005) 1-13.

39. M. Messner, S. V. Kurkov, P. Jansook, and T. Loftsson, Int. J. Pharm. 387 (2010) 199-208. 